\title{
Geometric and ergonomic characteristics of the uniportal video-assisted thoracoscopic surgery (VATS) approach
}

\author{
Luca Bertolaccini ${ }^{1}$, Andrea Viti ${ }^{1}$, Alberto Terzi $^{1}$, Gaetano Rocco ${ }^{2}$ \\ ${ }^{1}$ Surgery Unit, Sacro Cuore Don Calabria Research Hospital—Cancer Care Center, Negrar Verona, Italy; ${ }^{2}$ Division of Thoracic Surgery, Department \\ of Thoracic Surgical and Medical Oncology, IRCCS National Cancer Institute-Pascale Foundation, Naples, Italy \\ Correspondence to: Luca Bertolaccini, MD, PhD, FCCP. Thoracic Surgery Unit, Sacro Cuore Don Calabria Research Hospital—Cancer Care Center, \\ Via Don Angelo Sempreboni 5, 37024 Negrar Verona, Italy. Email: luca.bertolaccini@gmail.com.
}

\begin{abstract}
During the last three decades, video-assisted thoracoscopic surgery (VATS) has been revolutionizing the surgical treatment of several thoracic pathologies. Compared to open thoracotomy, VATS has demonstrated not only a significant reduction in pain, recovery time and complications but also a significant improvement in the post-operative quality of life for patients. The uniportal VATS technique was initially described in the early 2000s. This technique involves the simultaneous introduction of instruments parallel to a thoracoscope through one small incision, the breadth of a surgeon's finger, without further dissection of the intercostal space. Some papers have already demonstrated the advantage of uniportal VATS in comparison to the traditional three-port techniques in reducing postoperative pain, length of hospital stay and time of return to activities of daily living. Standard three-port VATS has a geometric configuration of a trapezoid that interferes with the optical source by creating a new optical plane which generates a torsion angle not favorable with standard two-dimensional monitors. By contrast, the uniportal VATS approach along a sagittal plane from a caudo-cranial perspective enables a projective plane that preserves the depth of intraoperative visualization. The instruments, as parallel lines from this plane, enable the surgeon to bring the operative fulcrum inside the chest. In addition, the uniportal VATS approach can significantly improve the surgeons' body posture during surgery since the surgeons can stand straight facing the monitor with minimal neck movement. Thus, the surgeons benefit from ergonomic advantages compared to the standard three-port approach.
\end{abstract}

Keywords: Uniportal video-assisted thoracoscopic surgery (uniportal VATS); ergonomics; lung cancer; minimally invasive thoracic surgery; video-assisted thoracoscopic surgery (VATS)

Submitted Oct 16, 2015. Accepted for publication Nov 21, 2015.

doi: 10.21037/acs.2015.12.05

View this article at: http://dx.doi.org/10.21037/acs.2015.12.05

\section{Introduction}

During the last three decades, video-assisted thoracoscopic surgery (VATS) has revolutionized the surgical approach to a number of thoracic pathologies (1). Compared to thoracotomy, VATS has demonstrated not only a significant reduction in pain, recovery time and complications but also an improvement in the post-operative quality of life for patients (2). Hence, VATS should be considered the conventional approach for almost all common thoracic operations (3). In the early 2000s, G.R. described the technique for uniportal VATS wedge pulmonary resection (4). Since this first report, progressive refinements of this technique were developed and applied to a broader range of surgical indications including major anatomic pulmonary resections for primary lung malignancy (5). Some papers have already demonstrated the advantage of uniportal VATS in comparison to the traditional three-port techniques in the reduction of postoperative pain, length of hospital stay and time of return to daily activities (6). Uniportal VATS involves the introduction of instruments parallel to a thoracoscope through one small incision wide 


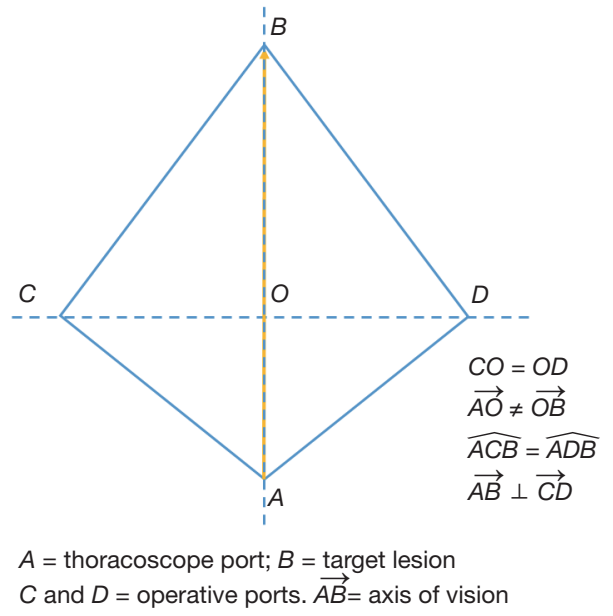

Figure 1 Ideal three-port VATS lobectomy settings. VATS, videoassisted thoracoscopic surgery.

as a surgeon's fingerbreadth without further dissection of the intercostal space. An enhanced hand-eye coordination to visualize and operate the thoracoscope and instruments is required. In particular, the thoracoscope is handled in order to visualize the position of the instruments at any time during the procedure with simple zooming in and out of the operative field (5). In this paper, we review the geometric and ergonomic characteristics of the uniportal VATS approach.

\section{Geometric characteristics of uniportal VATS}

The geometric configuration of the uniportal VATS approach is completely different to the standard three-port VATS settings. The use of a single port favors a translational approach of VATS instruments along a sagittal plane. The single port setting enables the instruments to move in the direction of two parallel lines approaching the target lesion from a cranio-caudal perspective. This allows the operative fulcrum to be brought inside the chest in a fashion similar to open surgery (1). The three-port VATS lobectomy approach typically uses small ports without rib spread. The strategy for port placement was described in the literature as a baseball diamond and resembles a trapezoid shape (Figure 1) (3). The surgeon's eyes (the thoracoscope) are at point $A$; the target lesion lies in front of the surgeon's eyes at point $B$; the other two ports are placed at points $C$ and $D$ to allow the left and right hand instruments to be placed and triangulated forwards towards the target at point $B$ along the two vectorial planes $\overrightarrow{C B}$ and $\overrightarrow{D B}$. The viewing axis

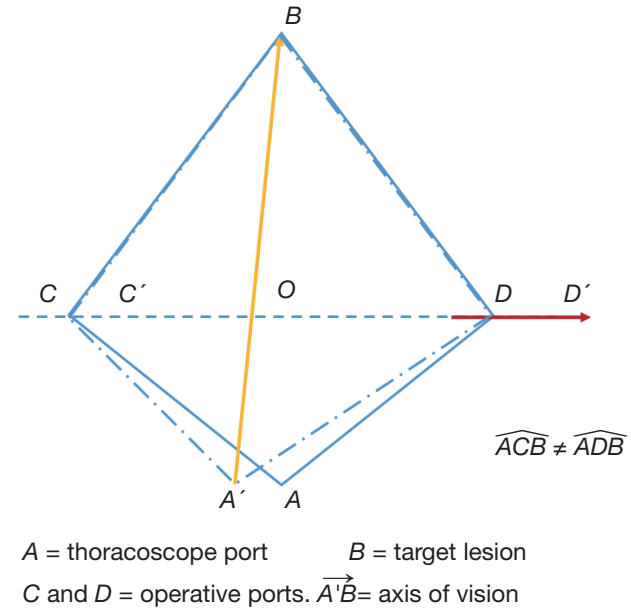

Figure 2 Real three-port VATS lobectomy settings. The real axis $\left(\overrightarrow{A^{\prime} B}\right)$ of the operation is translated posteriorly. VATS, videoassisted thoracoscopic surgery.

(vector $\overrightarrow{A B}$ ) is perpendicular to the operation port axis $C D$ and follows the natural longitudinal axis of the patient from the feet towards the head. Nevertheless, this setting fails to reproduce the real-life arrangement where the surgeons and assistants are situated around the operating table. In real settings, many surgeons stand anterior to the patient in the lateral decubitus position. Consequently, the real axis of the operation is translated posteriorly (Figure 2). The posterior port $(D)$ is translated along the viewing axis and the surgeon is too far to comfortably handle the instrumentation from their position. In addition, if the assistant stands on the opposite side of the operating table, the visual axis would be completely different. In order to cope with these technical and ergonomic challenges, the three-port VATS port placement strategy has been modified, as proposed by Henrik Hansen and coworkers, with the translation of the trapezoid (Figure 3) (7). The camera port $(A)$ has been brought more anterior to the anterior axillary line $\left(A^{\prime}\right)$ and the posterior port $(D)$ has been placed more caudally $\left(D^{\prime}\right)$. The utility port position $(C)$ remains unchanged. Although the trapezoid has been preserved, the axis $A^{\prime} B$ is more comfortable for the operating surgeon. The assistant stands on the same side of the operating table thus improving coordination. After further experience with the three-port VATS approach, it was realized that the posterior $3 \mathrm{~mm}\left(D^{\prime}\right)$ port was not always essential, resulting in a two-port VATS technique (Figure 4) (8). The three-port approach, whereby the trapezoid configuration allows maximal convergence of operative instruments from each side of the target lesion, 


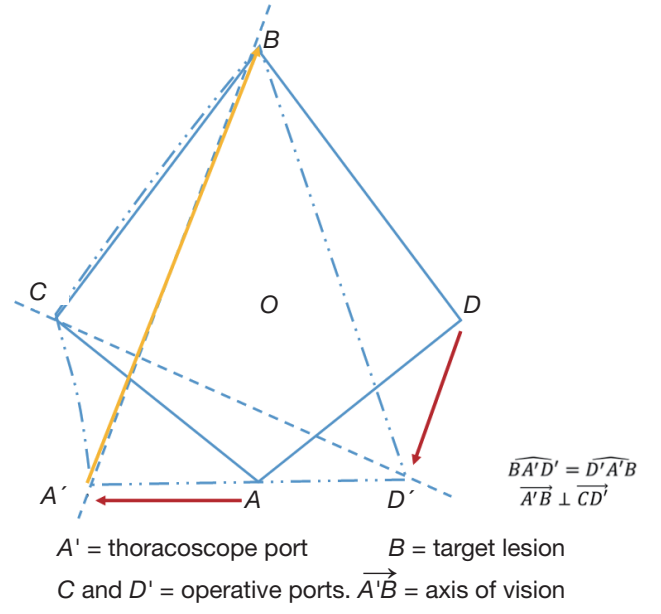

Figure 3 Anterior three-port VATS lobectomy settings. VATS, video-assisted thoracoscopic surgery.

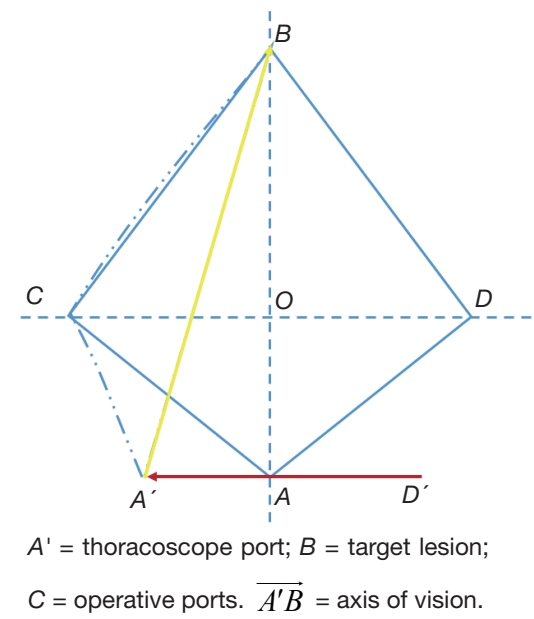

Figure 4 Two-port VATS lobectomy settings. VATS, videoassisted thoracoscopic surgery.

results in interference with the optical source (3). The uniportal approach requires translation of the thoracoscope and instruments $90^{\circ}$ along a sagittal plane passing from point $C$, allowing the operative instruments to target the lesion from a vertical and caudo-cranial perspective (Figure 5). To avoid mutual interference, the use of articulating instruments is of paramount importance for their ability to rotate the stem and jaws independently on different planes and with multiple angles. In fact, the approach to the target lesion in the lung is substantially similar to the approach that the surgeon would use in open surgery (9). In the uniportal VATS approach, the target lesion is located

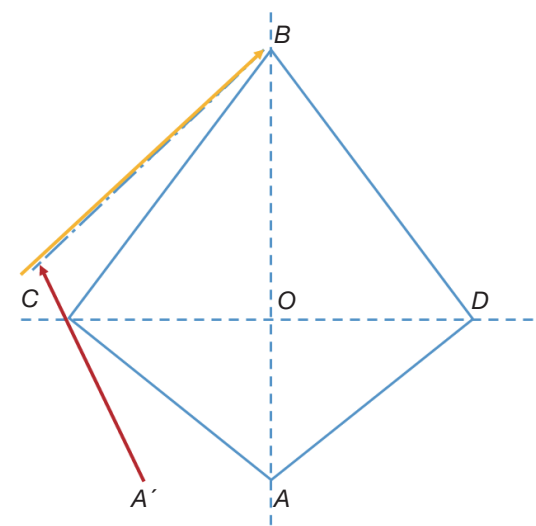

Figure 5 Uniportal VATS settings. The thoracoscope and instruments are translated $90^{\circ}$ along a sagittal plane passing from point $C$, bringing the operative instruments to address the target lesion from a vertical, caudo-cranial perspective. VATS, videoassisted thoracoscopic surgery.

in a projective plane with homogeneous coordinates. It is elevated with forceps perpendicularly from the parenchymal profile and resected by applying a stapler (or a curved clamp and overseen) at the base of this newly formed, coneshaped parenchymal area (10). The approach for a target lung lesion is then similar to a coaxial approach: surgeons work with their eyes and hands in the same plane (coaxial approach), much like open surgery and in contrast to threeport VATS (para-axial approach) (11).

\section{Ergonomic characteristics of uniportal VATS}

The VATS approach results in several ergonomic problems similar to laparoscopy and other minimally invasive surgical techniques. These relate to the large working area and rather static body position resulting in fatigue of the legs, extreme movements of the upper limbs and wrist as well as stiffness of the neck. The surgeon usually acts sideways from the patient during three-port VATS due to the position of the trocars which are fairly distant from the endoscope. The surgeon must rotate his or her torso and lean over the patient to operate, resulting in an unstable and tiring body position, which has to be maintained throughout the whole operation. In addition, the surgeon has to turn his or her neck and work in a different direction to the viewing direction of the monitor (12). In the uniportal VATS setup, the surgeon and the assistant should be positioned in front of the patient while in three-port access VATS the 
camera assistant could stand on the same or on the opposite side. Nevertheless, in our experience (13), the standing position of assistant surgeon in the three-port access suite was moved from the opposite side to the same side not only in major procedures but also in minor procedures to increase the assistant's skill. In the uniportal VATS setting, the surgeon and the assistant are placed in front of the patient so that they have the same field of vision and improved coordination and ergonomics (14). In the ErgonTrial (13), we chose to observe only thoracoscopic wedge resection since it is a frequently performed and relatively short procedure with technically standardized and clearly identifiable stages. Significant ergonomic benefits might be gained in the uniportal VATS environment suite as the viewing direction is brought back to the path orientation and restores the natural eye-hand-target axis. The physical workload, self-reported after performing uniportal VATS, is significantly less challenging with uniportal than with triportal VATS. The body posture during triportal VATS required more elbow flexion, causing prolonged use of the biceps muscle, and more wrist flexion, resulting in greater fatigue. In particular, due to instrument manipulation and interference from the surgical assistant, usually from his or her arm supporting the camera, the surgeon has to raise and abduct his shoulders, thus overloading the trapezius muscle. In contrast, the more neutral ergonomic posture during uniportal VATS enables manipulation without influencing instrument movements (13). Future research should evaluate the ergonomics in VATS major pulmonary resections investigating the effect of ergonomic interventions on physical and mental workload.

\section{Conclusions}

Standard three-port VATS has a trapezoidal configuration that interferes with the optical source by creating a new optical plane which generates a torsion angle not favorable with standard two-dimensional monitors. By contrast, the uniportal VATS approach to a lesion along a sagittal plane from a caudo-cranial perspective realizes a projective plane that preserves the depth of intraoperative visualization. The instruments, moving along parallel lines drawn from this plane, enable the surgeon to bring the operative fulcrum inside the chest. In addition, the uniportal VATS approach can significantly improve body posture during surgery since the surgeons can stand straight facing the monitor with minimal neck extension and rotation. This improved posture and more appropriate direction represent a substantial ergonomic advantage of uniportal VATS compared to the conventional triportal approach.

\section{Acknowledgements}

None.

\section{Footnote}

Conflicts of Interest: The authors have no conflicts of interest to declare.

\section{References}

1. Anile M, Diso D, Mantovani S, et al. Uniportal video assisted thoracoscopic lobectomy: going directly from open surgery to a single port approach. J Thorac Dis 2014;6:S641-3.

2. He J. History and current status of mini-invasive thoracic surgery. J Thorac Dis 2011;3:115-21.

3. Sihoe $\mathrm{AD}$. The evolution of minimally invasive thoracic surgery: implications for the practice of uniportal thoracoscopic surgery. J Thorac Dis 2014;6:S604-17.

4. Rocco G, Martin-Ucar A, Passera E. Uniportal VATS wedge pulmonary resections. Ann Thorac Surg 2004;77:726-8.

5. Gonzalez-Rivas D, Paradela M, Fernandez R, et al. Uniportal video-assisted thoracoscopic lobectomy: two years of experience. Ann Thorac Surg 2013;95:426-32.

6. Salati M, Rocco G. The uni-portal video-assisted thoracic surgery: achievements and potentials. J Thorac Dis 2014;6:S618-22.

7. Hansen HJ, Petersen RH, Christensen M. Videoassisted thoracoscopic surgery (VATS) lobectomy using a standardized anterior approach. Surg Endosc 2011;25:1263-9.

8. D'Amico T. Thoracoscopic lobectomy. J Med Sci 2007;27:95-100.

9. Bertolaccini L, Rocco G, Viti A, Terzi A. Geometrical characteristics of uniportal VATS. J Thorac Dis 2013;5:S214-6.

10. Gonzalez-Rivas D, Fieira E, Delgado M, et al. Is uniportal thoracoscopic surgery a feasible approach for advanced stages of non-small cell lung cancer? J Thorac Dis 2014;6:641-8.

11. Kamiyoshihara M, Igai H, Ibe T, et al. A 3.5-cm SingleIncision VATS Anatomical Segmentectomy for Lung Cancer. Ann Thorac Cardiovasc Surg 2015;21:178-82. 
12. Kranenburg L, Gossot D. Ergonomic problems encountered during video-assisted thoracic surgery. Minim Invasive Ther Allied Technol 2004;13:147-55.

13. Bertolaccini L, Viti A, Terzi A. Ergon-trial: ergonomic evaluation of single-port access versus three-port

Cite this article as: Bertolaccini L, Viti A, Terzi A, Rocco G. Geometric and ergonomic characteristics of the uniportal video-assisted thoracoscopic surgery (VATS) approach. Ann Cardiothorac Surg 2016;5(2):118-122. doi: 10.21037/ acs.2015.12.05 access video-assisted thoracic surgery. Surg Endosc 2015;29:2934-40.

14. Fieira Costa E, Delgado Roel M, Paradela de la Morena $\mathrm{M}$, et al. Technique of uniportal VATS major pulmonary resections. J Thorac Dis 2014;6:S660-4. 